be accounted for by changes in myocardial collagen content. However, qRT-PCR revealed that sarcoplasmic reticulum $\mathrm{Ca} 2+-$ ATPase (SERCA2a) expression was reduced in Hsd11b1fl/ flSm $22 \alpha$-Cre mice compared to controls. Furthermore, cardiomyocytes isolated from $11 \beta$-HSD1 deficient mice displayed a reduced rate of spontaneous contraction consistent with SERCA2a deficiency.

These data demonstrate that cardiovascular 11 $\beta$-HSD1 deficiency results in preserved systolic function with mild diastolic dysfunction in vivo. Altered $\mathrm{Ca} 2+$ handling within cardiomyocytes may impair relaxation and contribute to development of this phenotype.

\title{
CARDIOVASCULAR PHENOTYPING OF MICE WITH TARGETED 11ß-HYDROXYSTEROID DEHYDROGENASE TYPE 1 DELETION
}

doi:10.1136/heartjnl-2012-303148a.11

C I White, ${ }^{*}$ A Thompson, X Zhao, C Moran, K E Chapman, G A Gray. BHF/University Centre for Cardiovascular Science, Queen's Medical Research Institute, University of Edinburgh, 47 Little France Crescent, Edinburgh, EH16 4TJ

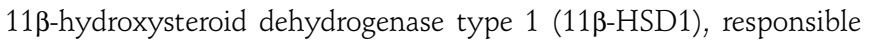
for intracellular regeneration of corticosteroids, is expressed widely in the cardiovascular system. This study aimed to determine the cardiovascular phenotype of mice in which 11ß-HSD1 was specifically deleted in cardiomyocytes and vascular smooth muscle cells by Cre-mediated recombination (Hsd11b1fl/flSm22 $\alpha$-Cre).

Western blotting and qRT-PCR confirmed loss of 11 -HSD1 only in cardiomyocytes and vascular smooth muscle cells of Hsd11b1fl/ flSm $22 \alpha$-Cre mice. Blood pressure, assessed by tail plesythmography, was similar in Hsd11b1fl/flSm22 $\alpha$-Cre and control (Hsd11b1fl/fl) littermates. High frequency ultrasound demonstrated that cardiac structure and systolic function were similar in both groups at 6, 10,20 and 35 weeks of age, with comparable end systolic and diastolic areas and ejection fractions. Consistent with this, post-mortem heart weight to body weight ratio was similar in both groups. Mitral valve pulsedwave Doppler ultrasound showed that E/A wave ratios were similar in Hsd11b1fl/flSm22 $\alpha$-Cre and controls. However, E wave deceleration was reduced, and deceleration time increased, in Hsd11b1fl/ flSm $22 \alpha$-Cre mice versus controls, suggesting mild diastolic dysfunction due to reduced compliance or relaxation deficit. This could not 